\title{
Hybrid capture II for high-risk human papillomavirus DNA testing to detect cervical precancerous lesions: A qualitative and quantitative study
}

\author{
LI MA, MEI-LU BIAN, JIAO-YING CHENG, WEI XIAO, MIN HAO, JUAN ZHU, YING CHEN and JUN LIU
}

Department of Gynecology and Obstetrics, China-Japan Friendship Hospital, Beijing 100029, P.R. China

Received August 5, 2009; Accepted October 9, 2009

DOI: 10.3892/etm_00000031

\begin{abstract}
Hybrid capture II (HC-II) is the only technique that can be used in clinical human papillomavirus (HPV) DNA detection. However, there is controversy in regards to how to analyze and assess the viral load of high-risk (HR)-HPV by use of HC-II and the relation between viral load and cervical lesions. In this study, we analyzed the results of a sequential screening of outpatients at the Department of Obstetrics and Gynecology of the China-Japan Friendship Hospital, and we aimed to explore the relationship between HR-HPV viral load and the severity of cervical lesions, and to clarify the clinical significance of the titer of HR-HPV DNA determined by HC-II. Using HC-II, 2,761 women were screened for HR-HPV DNA combined with cytological testing using liquid-based cytology. All women with HR-HPV-positive results or abnormalities in cytology underwent a cervical biopsy through colposcopy. Cervical biopsies were taken in 1,051 women. The HR-HPV infection rate was $78.35 \%$ (76/97) in HPV-associated lesions, $87.33 \%$ (193/221) in cervical intraepithelial neoplasia (CIN) I, 94.74\% (144/152) in CIN II, 100\% (178/178) in CIN III and $100 \%(20 / 20)$ in invasive cervical cancer (ICC), respectively $(\mathrm{P}<0.05)$. Based on the criteria of histopathology, the sensitivity of HR-HPV DNA testing by HC-II for detecting high-grade cervical lesions was $97.71 \%$, the specificity was $79.64 \%$, the positive-predictive value was $41.06 \%$ and the negativepredictive value was $99.59 \%$. The viral loads of HR-HPV DNA were $512.15 \pm 764.19$ in HPV-associated lesions, 753.95 \pm 978.27 in CIN I, 871.08 \pm 1003.52 in CIN II, 603.40 \pm 740.25 in CIN III and $466.44 \pm 673.05$ in ICC, respectively. In conclusion, the positive rate of HR-HPV increased significantly in accordance with the severity of cervical lesions. The viral loads of cervical inflammatory lesions were markedly lower than CINs and
\end{abstract}

Correspondence to: Dr Mei-Lu Bian, Department of Gynecology and Obstetrics, China-Japan Friendship Hospital, Beijing 100029, P.R. China

E-mail: bianmeilu@hotmail.com

Key words: human papillomavirus, cervical intraepithelial neoplasia, viral load
ICC. The viral loads of HR-HPV DNA tested by HC-II had no correlation with the grade of cervical lesions.

\section{Introduction}

Since hybrid capture II (HC-II) was approved by the US Food and Drug Administration (FDA) in 1999, it has been utilized in China for nearly 10 years. At present, $\mathrm{HC}$-II is the most advanced viral DNA detection technique, and also the only technique used in clinical human papillomavirus (HPV) DNA detection. Epidemiologic studies have shown that cervical cancer is an infectious disease. Persistent infection of high-risk (HR)-HPV is a necessary condition of cervical cancer and precancerous lesions - cervical intraepithelial neoplasia (CIN) (1). HPV DNA detection by use of HC-II has played an important role in the screening of cervical cancer and CINs (2). The application of HC-II has reached a consensus in some clinical fields: i) combined use with liquid-based cytology (LBC) in cervical cancer screening to improve sensitivity and specificity; ii) use in a triage of patients with atypical squamous cells of unknown significance or more severe cytological lessions; iii) use in the follow-up of the patients with CIN II or more severe histological lessions. However, there is controversy as to how to analyze and assess the viral load of HR-HPV by use of HC-II and the relation between viral load and cervical lesions. At present, there are three viewpoints. i) Viral loads of HR-HPV DNA increase with the severity of cervical neoplasias $(3,4)$. ii) Both cervical cancer and CINs are highly influenced by HR-HPV viral loads (5). iii) From cervical inflammation to CINs, the titer of the HC-II test does not demonstrate a clear line, but the higher the titer, the greater possibility of high-grade squamous intraepithelial lesions (6). In this study, we analyzed the results of a sequential screening of outpatients at the Department of Obstetrics and Gynecology of the China-Japan Friendship Hospital, and we aimed to explore the relationship between HR-HPV viral load and the severity of cervical lesions, and to clarify the clinical significance of the titer of HR-HPV DNA determined by HC-II.

\section{Materials and methods}

Study population. From September 2006 to March 2009, 2,761 women who visited the Department of Obstetrics and 
Gynecology of the China-Japan Friendship Hospital consented to cervical cancer screening using HC-II and LBC. Written informed consent was obtained from all patients. All of the cases met the following criteria: defined sexual behavior, no current pregnancy, no previous hysterectomy, and no previous ablative or excisional therapy of the cervix. The mean age of the 2,761 women was $39.25 \pm 10.08$ years of age (range, 17-80 years).

Cytology. A sample of drop epidermic cells from the ectocervix and endocervix was obtained by performing complete rotations with a cervical cytobrush and immediately immersed in fixative solution. Thin-layer cytology specimens were obtained using the Surepath liquid-based cytology and Thinprep liquid-based cytology tests. Cytologic diagnosis was made according to the cervical cytology criteria of the 2001 Bethesda System (7).

HPV DNA testing using hybrid capture II. HR-HPV DNA detection was performed using the automated HC-II test system (Qiagen, Gaithersburg, MD, USA). The samples were analyzed for the 13 most common HR-HPV DNA types: 16 , $18,31,33,35,39,45,51,52,56,58,59$ and 68. Specimens were considered positive for HPV infection when the relative lighting unit (RLU) value was equal to or greater than the positive control (PC), which corresponds to $1 \mathrm{ng} / \mathrm{l}$ of HPV DNA ( 4700 copies of the HPV genome/ml assay).

Colposcopy and biopsy. Colposcopic examination of the cervix was performed in women with either HR-HPV-positive results or abnormalities in cytology, or both. Negative results in both cytology and HR-HPV testing predicted a low risk of cervical neoplasia. A punch biopsy was taken from key sites of acetowhite epithelium, mosaic, punctation, leukoplakia and atypical vessels that were represented after daubing with $5 \%$ acetum. If no abnormal lesion was found, a punch biopsy was taken from positions 2, 4, 8 and 10 o'clock of the cervix according to routine biopsy methods. Endocervical canal curettage was performed when colposcopic examination was unsatisfactory. The patients with high-grade lesions accepted loop electrosurgical excision procedure (LEEP), or cold knife conization (CKC), or even hysterectomy for further diagnosis and treatment. The most severe diagnosis was determined as final histopathological diagnosis. The diagnostic criteria of high-grade lesions were defined as equal to or more severe than CIN II.

Statistical analysis. Based on the criteria of histopathology, the sensitivity, specificity, positive-predictive value and negative-predictive value were calculated. The difference of mean data was estimated by the t-test, and HPV test results and final histologic diagnoses were analyzed by the $\chi^{2}$ test. Based on the quartiles of the logarithms of RLU/PC, the positive cases of HR-HPV DNA were divided into 3 groups: low-degree loads $\left(\log _{10} \mathrm{RLU} / \mathrm{PC}=0-1.73\right)$, moderate-degree loads $\left(\log _{10} \mathrm{RLU} / \mathrm{PC}=1.74-2.78\right)$ and high-degree loads $\left(\log _{10}\right.$ RLU/PC $\left.=2.79-3.73\right)$. The patients with negative HPV $\left(\log _{10}\right.$ RLU/PC $\left.<0\right)$ were considered as the normal control group. Statistical analysis software SPSS 13.0 was used. $\mathrm{P}<0.05$ was considered statistically significant.

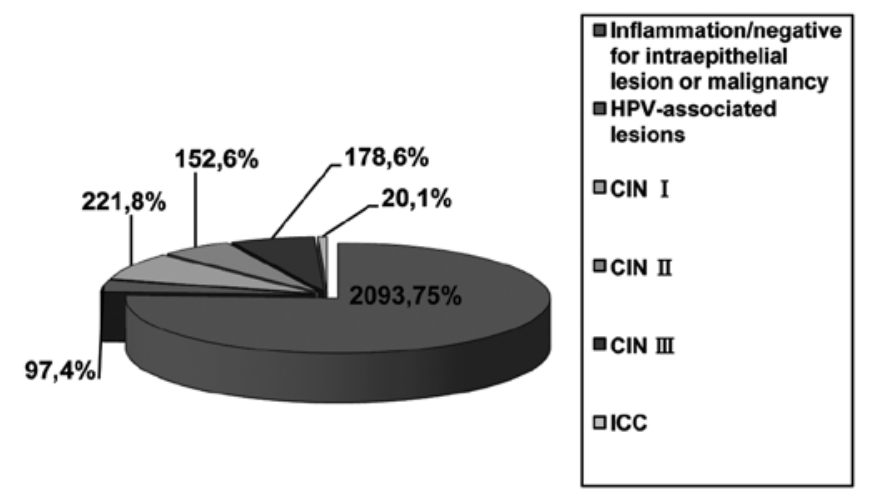

Figure 1. The histopathological diagnoses in 2,761 cases.

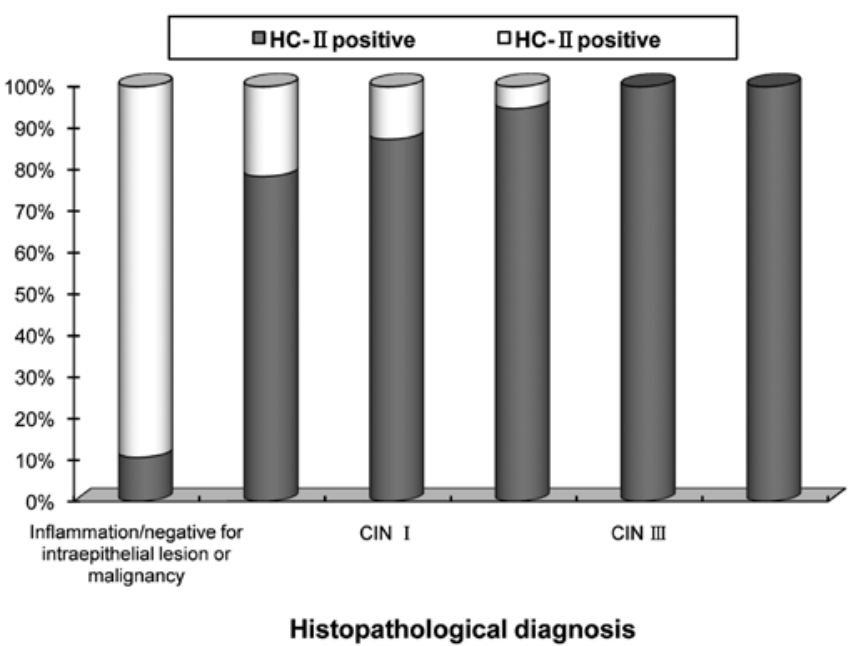

Figure 2. HR-HPV infection in different cervical lesions.

\section{Results}

Histopathological results. Cervical biopsies were obtained upon consent in 1,051 women by colposcopy. HPV-associated lesions implied transient HPV infection in the uterine cervix. Final pathological diagnoses included HPV-associated lesions in 97 cases, CIN I in 221 cases, CIN II in 152 cases, CIN III in 178 cases, invasive cervical cancer (ICC) in 20 cases and inflammation in 383 cases, respectively. A total of 1,710 women both LBC- and HR-HPV-negative had an extremely low-risk to develop high-grade CINs during the next several years (8). We considered them to be negative for intraepithelial lesions or malignancy (Fig. 1).

Age analysis. The distribution of patient age with different histopathological diagnoses is shown in Table I.

Morphological analysis. Pathological change with kiolocytosis was found in $76.92 \%$ (170/221) of CIN I, 65.13\% (99/152) of CIN II and $38.20 \%$ (68/178) of CIN III cases, respectively $\left(\chi^{2}=63.621, \mathrm{P}<0.01\right)$. Gland involvement in the cervix was found in $3.17 \%$ (7/221) of CIN I, 23.03\% (35/152) of CIN II and $74.72 \%(133 / 178)$ of CIN III cases, respectively $\left(\chi^{2}=240.281\right.$, $\mathrm{P}<0.01)$. 
Table I. Age and cervical lesions.

\begin{tabular}{|c|c|c|c|c|}
\hline Histopathological diagnosis & Mean \pm SD & Age range & No. & P-value \\
\hline $\begin{array}{l}\text { Inflammation/negative for } \\
\text { intraepithelial lesion or malignancy }\end{array}$ & $39.63 \pm 10.36$ & $17-80$ & 2093 & \\
\hline HPV-associated lesions & $37.68 \pm 9.03$ & $23-74$ & 97 & $0.041^{\mathrm{a}}$ \\
\hline CIN I & $36.61 \pm 9.38$ & $20-59$ & 221 & \\
\hline CIN II & $38.45 \pm 9.01$ & $19-65$ & 152 & \\
\hline CIN III & $39.13 \pm 8.52$ & $20-71$ & 178 & \\
\hline ICC & $41.24 \pm 12.41$ & $29-60$ & 20 & $0.043^{\mathrm{b}}$ \\
\hline
\end{tabular}

at-test, 'Inflammation/negative for intraepithelial lesion or malignancy' vs. 'HPV-associated lesions', p<0.05. bt-test, 'CIN III' vs. ‘ICC', p<0.05. HPV, human papillomavirus; CIN, cervical intraepithelial neoplasia; ICC, invasive cervical cancer.

Table II. Range of RLU/PC in different cervical lesions.

\begin{tabular}{lcrr}
\hline Histopathological diagnosis & RLU/PC (mean \pm SD) & RLU/PC range & Median RLU/PC \\
\hline Inflammation/negative for & $424.26 \pm 721.19$ & $1.02-5399.48$ & 59.46 \\
intraepithelial lesion or malignancy & & & 222 \\
HPV-associated lesions & $512.15 \pm 764.19$ & $1.03-3268.43$ & 76 \\
CIN I & $753.95 \pm 978.27$ & $1.09-5387.61$ & 193 \\
CIN II & $871.08 \pm 1003.52$ & $1.05-3884.58$ & 276.37 \\
CIN III & $603.40 \pm 740.25$ & $1.39-3743.53$ & 448.52 \\
ICC & $466.44 \pm 673.05$ & $1.48-2414.92$ & 272.86 \\
\hline
\end{tabular}

RLU, relative lighting unit; PC, positive control; HPV, human papillomavirus; CIN, cervical intraepithelial neoplasia; ICC, invasive cervical cancer.

Results of hybrid capture II test. Positive HR-HPV DNA was found in $30.17 \%(833 / 2761)$ of all cases. The positive rates were $78.35 \%(76 / 97)$ in HPV-associated lesions, $87.33 \%$ $(193 / 221)$ in CIN I, $94.74 \%$ (144/152) in CIN II, 100\% (178/178) in CIN III and $100 \%(20 / 20)$ in ICC cases, respectively $\left(\chi^{2}=46.781, \mathrm{P}<0.01\right)$, while it was only $10.61 \%(222 / 2093)$ in inflammation/negative for intraepithelial lesion or malignancy cases (Fig. 2).

Analysis of viral loads. The viral loads of HR-HPV DNA tested by HC-II are listed in Table II. The median $\log _{10} \mathrm{RLU} /$ $\mathrm{PC}$ valuewas 1.77 in inflammation/negative for intraepithelial lesion or malignancy cases, 1.83 in HPV-associated lesions, 2.44 in CIN I, 2.65 in CIN II, 2.44 in CIN III and 2.13 in ICC cases, respectively. The distribution of viral loads of HR-HPV DNA in different cervical lesions is shown in Fig. 3.

The positive cases were divided into 3 groups: low-degree loads, moderate-degree loads and high-degree loads, respectively. The distributions of viral loads in different cervical lesions are shown in Table III.

The differences in HR-HPV viral load distribution in different cervical lesions were statistically significant $\left(\chi^{2}=57.957, \mathrm{P}<0.01\right)$. There was no statistical significance between inflammation/negative for intraepithelial lesion or malignancy cases and HPV-associated lesions $\left(\chi^{2}=1.231\right.$, $\mathrm{P}=0.540)$. Also, there was no statistical significance between
CINs and ICC (CIN I vs. CIN II, $\chi^{2}=1.212, \mathrm{P}=0.545$; CIN II vs. CIN III, $\chi^{2}=5.592, \mathrm{P}=0.061$; CIN III vs. ICC, $\chi^{2}=2.155$, $\mathrm{P}=0.340)$. However, the difference between HPV-associated lesions and CIN I was statistically significant $\left(\chi^{2}=10.974\right.$, $\mathrm{P}=0.004)$.

The range of RLU/PC values tested by HC-II was widely distributed from one to thousands in different cervical lesions. When the data were transformed to logarithms, there was no statistical significance between CINs and ICC. This implied that the viral loads of HR-HPV DNA had no correlation with the severity of the cervical lesions. In inflammation/ negative for intraepithelial lesion or malignancy cases and HPV-associated lesions, the viral loads of HR-HPV were low-degree loads when analyzed by the median RLU/PC, or analyzed by the Chi-square test among different groups. But there was statistically significant difference between moderate-degree loads in CINs and high-degree loads in ICC.

The data showed that the positive HR-HPV rate in women $>40$ year of age was the highest (43.46\%). But there was no statistical significance between the viral loads of HR-HPV and patient age $\left(\chi^{2}=3.968, \mathrm{P}=0.410\right)$ (Table IV).

\section{Discussion}

HPV infection and cervical intraepithelial neoplasias. Based on their association with cervical cancer and precursor 


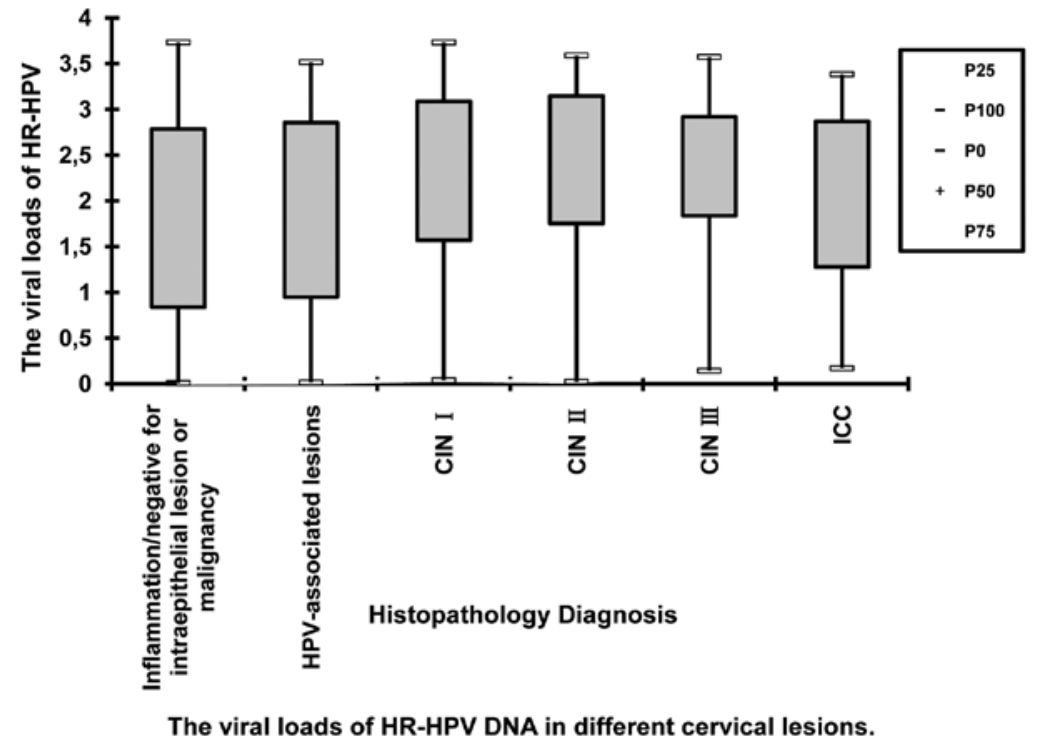

Figure 3. Viral loads of HR-HPV DNA in different lesions.

Table III. The distributions of viral loads of HR-HPV in different cervical lesions.

\begin{tabular}{lcrr}
\hline & \multicolumn{3}{c}{$\log _{10}$ RLU/PC } \\
\cline { 2 - 4 } & $0.00-1.73$ & $1.74-2.78$ & $2.79-3.73$ \\
\hline Inflammation/negative for & $107(48.20)$ & $59(26.58)$ & $56(25.22)$ \\
intraepithelial lesionor malignancy (\%) & $37(48.69)$ & $16(21.05)$ & $23(30.26)$ \\
HPV-associated lesions (\%) & $54(27.98)$ & $67(34.71)$ & $72(37.31)$ \\
CIN I (\%) & $35(24.31)$ & $47(32.64)$ & $62(43.05)$ \\
CIN II (\%) & $37(20.78)$ & $81(45.51)$ & $60(33.71)$ \\
CIN III (\%) & $7(35)$ & $7(35)$ & $6(30)$ \\
ICC (\%) & & 144 \\
\hline
\end{tabular}

HR, high risk; HPV, human papillomavirus; RLU, relative lighting unit; PC, positive control; CIN, cervical intraepithelial neoplasia; ICC, invasive cervical cancer.

Table IV. Age and viral loads of HR-HPV DNA.

\begin{tabular}{|c|c|c|c|c|}
\hline \multirow[b]{2}{*}{$\log _{10}$ RLU/PC } & \multicolumn{3}{|c|}{ Age } & \multirow[b]{2}{*}{ Total $(\%)$} \\
\hline & $<30(\%)$ & $30-40(\%)$ & $>40(\%)$ & \\
\hline $0.00-1.73$ & $54(19.49)$ & $92(33.22)$ & $131(47.29)$ & $277(33.25)$ \\
\hline $1.74-2.78$ & $53(19.13)$ & $103(37.18)$ & $121(43.69)$ & $277(33.25)$ \\
\hline $2.79-3.73$ & $63(22.58)$ & $106(37.99)$ & $110(39.43)$ & $279(33.50)$ \\
\hline Total & $170(20.41)$ & $301(36.13)$ & $362(43.46)$ & 833 \\
\hline
\end{tabular}

HR, high risk; HPV, human papillomavirus; RLU, relative lighting unit; PC, positive control; CIN, cervical intraepithelial neoplasia; ICC, invasive cervical cancer.

lesions, HPV may be divided into high-risk (HR)-HPV and low-risk (LR)-HPV types. LR-HPV infection often results in condyloma acuminatum of the reproductive organs and low-grade lesions of the cervix. It often has obvious features of morphology. The classical characteristic is appearance of kiolocytosis in malpighian epithelium. It is characterized 
by karyomegaly, nuclear enlargement with binucleation, irregularities in the nuclear membrane and hyperchromasia. Perinucleus cavity may be found, and kiolocytosis has diagnostic significance. But HR-HPV infection may be short of morphological evidence. If HR-HPV infection continues for 8-10 years, high-grade CIN (CIN II and III) may occur, and invasive cervical cancer may progress within 5-10 years. Some studies revealed that HPV infection exists in $99.80 \%$ of cervical cancer cases (9).

In this study, the morphological concomitance of kiolocytosis was $76.92 \%$ in CIN I, $65.13 \%$ in CIN II and $38.20 \%$ in CIN III cases, respectively. This implied that LR-HPV infection decreases significantly as the CIN grade progresses. However, in our study positive HR-HPV tested by HC-II was $87.33 \%$ in CIN I, $94.74 \%$ in CIN II and $100 \%$ in CIN III cases, respectively. This implied that the infection of HR-HPV increases as the lesion progresses. These results prove that LR-HPV infection is correlated with low-grade lesions (CIN I), while HR-HPV infection is necessary for high-grade lesions (CINs II and III) and cervical cancer. HR-HPV infection played a major role in the occurrence and progression of cervical cancer. Morphological study showed that with the progression of CINs, cervical gland involvement predominantly increased. This demonstrated that invasion of high-grade lesions might increase, and these lesions should be treated more actively.

Relationship between the viral loads and cervical intraepithelial neoplasias and invasive cervical cancer. The range of RLU/PC values was widely distributed in this study from one to thousands including the normal cervix, inflammation of the cervix, CINs and ICC. Thus, the viral loads could not be used to define whether the lesion was benign or malignant or its severity. To investigate the relation between viral loads and lesions, RLU/PC values were transformed into logarithms and categorized into four groups. It was found that there was no statistical significance between inflammation/ negative for intraepithelial lesion or malignancy cases and HPV-associated lesions, and the viral loads of these groups were at a low level. The $\log _{10}$ RLU/PC values were 1.77 and 1.83, and this level was lower than those of CINs and ICC. The viral loads of CINs and ICC were at a moderate or high level. There was no relationship between the viral loads and the severity of cervical lesions. In this study $\log _{10} \mathrm{RLU} / \mathrm{PC}$ values of CINs were higher than that of ICC. This might have been partly due to the relatively fewer cases of ICC. However, the median $\log _{10}$ RLU/PC value for CIN I was the same as that for CIN III; both were 2.44 and lower than that in CIN II (2.65). This showed that the $\log _{10}$ RLU/PC value does not reflect the severity of cervical lesions. On the other hand, due to the limitations of histopathology, the actual lesion sample was at times not acquired by biopsy and might induce positive results for HC-II with negative results for histopathology in some cases.

Clinical significance of HR-HPV DNA testing using hybrid capture II. The threshold value of RLU/PC is defined by 1.0. But RLU/PC $<1.0$ does not mean absence of HPV infection. RLU/PC $<1.0$ means $4700 \mathrm{HPV}$ copies/ml. According to this threshold value, we could optimally evaluate the sensitivity and specificity for the risk of high-grade cervical lesions. Some studies showed $(8,10)$ that better sensitivity and specificity could not be obtained by increasing the threshold value of RLU/PC. Therefore, HR-HPV DNA tested by $\mathrm{HC}$-II had clinical significance at RLU/PC $\geq 1$. Based on the histopathology in this study, the sensitivity of HR-HPV DNA tested by HC-II for detecting high-grade cervical lesions was $97.71 \%$, the specificity was $79.64 \%$, the positive-predictive value was $41.06 \%$, and the negative-predictive value was $99.59 \%$, respectively. A vast amount of data in China and other countries revealed that the main advantage of HR-HPV DNA detection is its extremely high negative-predictive value (11-15). It is possible to infer that individuals with negative HR-HPV have an extremely low risk of cervical cancer. In this study, the sensitivity and negative-predictive value were very high. Thus, as HC-II is a simple accessible method, we can apply it independently for the screening of cervical cancer and precancerous lesions. In our study, patients in the CIN I group were the youngest in age, and the range of RLU/PC values was widely distributed. This implied that the HPV virus might be in a free state in infected young women. The virus might be duplicated temporarily in a large amount, but might be eliminated by the host through the autoimmunity mechanism. High-grade cervical lesions would occur only when the viral DNA integrated with the host DNA. Studies have shown that persistent HR-HPV infection is a necessary condition of CINs and cervical cancer (16). LEEP and CKC have become routine treatment for CINs, but lesions may recur after clinical treatment. How to monitor cases with persistent HR-HPV infection and follow up cases after treatment is still unclear. Yet, HC-II testing offers a reliable method for the management and follow-up after treatment of cervical precancerous lesions.

In conclusion, the viral loads of HR-HPV DNA tested by HC-II had no correlation with the severity of cervical lesions. The viral load of inflammatory cervical lesions was markedly lower than those of CINs and ICC. The positive rate of HR-HPV increased significantly with the progression of cervical lesions. HC-II testing has important value both in the screening of cervical lesions and in the management and follow-up after treatment of cervical precancerous lesions.

\section{References}

1. Bosch FX, Manos MM, Munoz N, Sherman M, Jansen AM, Peto J, Schiffman MH, Moreno V, Kurman R and Shah KV: Prevalence of human papillomavirus in cervical cancer: a worldwide perspective. International Biological Study on Cervical Cancer (IBSCC) Study Group. J Natl Cancer Inst 87: 796-802, 1995.

2. Ying $\mathbf{J}$ and Lingya $P$ : Role of high-risk human papillomavirus testing in the screening and management of cervical cancer precursors. Acta Acade Med Sinic 29: 691-696, 2007.

3. Zhihong H, Deying Q, Ding W, Danhua H, Minjian C and Yanhong SH: Study of the relationship between loads of human papilloma virus in cervical carcinoma and cervical intraepithelial neoplasia. Matern Child Health Care Chin 21: 1557-1559, 2006.

4. Shumin L, Wenhua ZH, Lingying W, Fanghui ZH, Manni H, Nan L and Feng CH: Preliminary study on the relationship between loads of human papillomavirus in cervical carcinoma and cervical intraepithelial neoplasia. Chin J Obstet Gynecol 39: 400-402, 2004.

5. Fanghui ZH, Junfei M, Youlin Q, Shoude R, Ling L and Wenhua ZH: Association between high-risk human papillomavirus DNA load and cervical intraepithelial lesion. Chin J Epidemiol 25: 921-924, 2004. 
6. Li J, Jinghe L, Youfang $\mathrm{W}$ and Xuemei CH: Relationship of HR-HPV DNA loads with the stages of CIN. Reprod Contrac 26 : 422-425, 2006.

7. Apgar BS, Zoschnick L and Wright TC Jr: The 2001 Bethesda System terminology. Am Fam Physician 68: 1992-1998, 2003.

8. Syrjanen S, Shabalova IP, Petrovichev N, Kozachenko VP, Zakharova T, Paianidi J, Podistov JI, Chemeris G, Sozaeva LG, Lipova EV, Tsidaeva I, Ivanchenko OG, Pshepurko AA, Zakharenko S, Nerovina R, Kljukina LB, Erokhina OA, Branovskaja MF, Nikitina M, Grunberga V, Grunberg A, Juschenko A, Tosi P, Cintorino M, Santopietro R and Syrjanen KJ: Human papillomavirus testing and conventional Pap smear cytology as optional screening tools of women at different risks for cervical cancer in countries of the former Soviet Union. J Low Genit Tract Dis 6: 97-110, 2002.

9. Tarkanen J, Auvinen E, Nieminen P, Malmi R,Vartianen J, Timonen T, Laurila P, Raisanen I, Unnerus HA, Sakki A, Mattila P, van Den, Brule AV and Tapper AM: HPV DNA testing as an adjunct in the management of patients with low-grade cytological lesions in Finland. Acta Obstet Gynecol Scand 86: 367-372, 2007.

10. Ordi J, Alonso I, Torne A, Esteve R, Sierra E, Campo E and Puiq-Tintore LM: Human papillomavirus load in Hybrid Capture II assay: does increasing the cutoff improve the test? Gynecol Oncol 99: 313-319, 2005.

11. Yufei $\mathrm{C}$ and Guangze $\mathrm{ZH}$ : The roles of high-risk human papillomavirus detection in cervical cancer screening. Matern Child Health Care Chin 21: 3434-3436, 2006.
12. Sherman ME, Lorincz AT, Scott DR, Wacholder S, Castle PE, Glass AG, Mielzynska-Lohnas I, Rush BB and Schiffman M: Baseline cytology, human papillomavirus testing, and risk for cervical neoplasia: a 10-year cohort analysis. J Natl Cancer Inst 95: 46-52, 2003

13. Cuzick J, Szarewski A, Cubie H, Hulman G, Kitchener H, Luesley D, McGoogan E, Menon U, Terry G, Edwards R, Brooks C, Desai M, Gie C, Ho L, Jacobs I, Pickles C and Sasieni P: Management of women who test positive for high-risk types of human papillomavirus: the HART study. Lancet 362: 1871-1876, 2003.

14. Clavel C, Cucherousset J, Lorenzato M, Caudroy S, Nou JM, Nazeyrollas P, Polette M, Bory JP, Gabriel R, Ouereux C and Birembaut P: Negative human papillomavirus testing in normal smears selects a population at low risk for developing high-grade cervical lesions. Br J Cancer 90: 1803-1808, 2004.

15. Deying Q, Jianmin C, Ding W, Renhai Z, Aihua L, Yanhong SH, Danhua $\mathrm{H}$ and Zhihong $\mathrm{H}$ : Combining high-risk human papillomavirus DNA test and cytological test to detect early cervical dysplasia. Chin J Obstet Gynecol 41: 34-37, 2006.

16. Munoz N, Bosch FX, Sanjose SDE, Herrero R, Castellsaque X, Shah KV, Snijder PJ, Meijer CJ and the International Agency for Research on Cancer Multicenter Cervical Cancer Study Group: Epidemiologic classification of human papillomavirus types associated with cervical cancer. N Engl J Med 348: 518-527, 2003. 